\title{
Nuclear data for medical applications: an overview
}

\author{
By S. M. Qaim* \\ Institut für Nuklearchemie, Forschungszentrum Jülich GmbH, D-52425 Jülich, Germany
}

(Received June 19, 2000; accepted in revised form September 26, 2000)

\section{Nuclear reaction / Cross section / Decay data / Diagnosis / Therapy / Data files}

\begin{abstract}
Summary. A brief introduction to nuclear data in medicine is given. The choice of a radioisotope for medical application demands an accurate knowledge of radioactive decay data. Short-lived single photon and $\beta^{+}$-emitters are preferred for diagnostic investigations, and longer-lived corpuscular radiation emitting radioisotopes for endoradiotherapy. The nuclear reaction cross section data, on the other hand, are needed for optimising the production routes. Besides radioactive isotopes, the use of ionising radiation in therapy is discussed. External radiation therapy has achieved an important place in medicine. The role of nuclear data is briefly discussed; they are needed for radiation dose calculations. The hitherto rather neglected activation products in proton therapy are considered. The methodology of development of a nuclear data file for medical applications is outlined.
\end{abstract}

\section{Introduction}

Soon after the discovery of radioactivity by $\mathrm{H}$. Becquerel in 1896, thoughts started developing about a possible application of this phenomenon in medicine. It was George de Hevesy, a Hungarian born physical chemist, who first introduced radioactive tracers in biology. This concept was followed by several other scientists. Tracer applications in general, and nuclear medicine in particular, received an impetus through the development of nuclear reactors and their use in the production of a large number of radioisotopes. The availability of organic compounds labelled with $\beta^{-}$-emitting radioisotopes like ${ }^{3} \mathrm{H},{ }^{14} \mathrm{C},{ }^{32} \mathrm{P},{ }^{35} \mathrm{~S},{ }^{125} \mathrm{I}$, etc. contributed appreciably to the development of biochemistry via in vitro methods. Also for in vivo investigations a variety of radiopharmaceuticals labelled with $\gamma$-emitting radioisotopes like ${ }^{18} \mathrm{~F},{ }^{51} \mathrm{Cr},{ }^{99 \mathrm{~m}} \mathrm{Tc},{ }^{131} \mathrm{I}$, etc. were introduced. However, for many diagnostic studies, neutron deficient radioisotopes appeared to be more suitable. Continuous efforts have therefore been underway to develop cyclotrons and accelerators devoted to the production of those radioisotopes. Today, the radioisotope production technology is well developed and both reactors and cyclotrons are utilized for production purposes. The proper use of nuclear data constitutes an integral part of any production process. The field of diagnostic nuclear medicine (i.e. diagnostic studies using radioactive tracers) is flourishing [cf. 1,2] and demands new radioisotopes

\footnotetext{
*E-mail: s.m.qaim@fz-juelich.de
}

and radiopharmaceuticals for investigating various biochemical phenomena.

Radioactivity is unique in its properties since it can contribute to both diagnosis and therapy, the former by virtue of its high detection sensitivity and the latter through the biological effects of radiation. Depending on its decay characteristics and use, a radioisotope can therefore be classified as a diagnostic or a therapeutic radionuclide. The therapeutic radioisotopes, especially those suitable for endoradiotherapy, have been gaining enhanced attention in recent years [cf. 1,2]. There is considerable need of nuclear data research relevant to the production and application of those radioisotopes.

In addition to radioactive isotopes, ionising radiation in general has found many applications in therapy. Besides photons, electrons and neutrons, high-energy charged particles like protons, ${ }^{4} \mathrm{He}$ and heavy ions $\left({ }^{14} \mathrm{~N},{ }^{22} \mathrm{Ne}\right.$, etc.) have been finding increasing use in the treatment of various types of tumours and malignant diseases. The data needs in this regard are extensive. Also modelling of radiation transport is important to be able to estimate the radiation dose properly.

In this introductory article the role of nuclear data in both diagnosis and therapy is outlined. The discussion is grouped together under "radioisotope programmes" and "external radiation therapy". Finally, the development of a nuclear data file for medical applications is considered.

\section{Significance of nuclear data in medically related radioisotope programmes}

In nuclear medicine programmes involving radioisotopes, both radioactive decay and nuclear reaction cross section data are needed. We briefly discuss the two types of data below.

\section{Radioactive decay data}

These data allow a calculation of the radiation dose to the patient caused by the administered radioactivity. The methodology used throughout the world for internal dose calculations is provided by the Medical Internal Radiation Dose Committee (MIRD) of the Society of Nuclear Medicine in the United States and its many collaborators and consultants, and is referred to as the "MIRD formalism". According to this formalism the dose is determined via the expression:

$$
\bar{D}=2.13 \bar{c} \sum_{i} n_{i} \bar{E}_{i} \Phi_{i},
$$

where 
$\bar{c}$ is the cumulative concentration of activity $\left(\mathrm{Bq} \cdot \frac{T_{\mathrm{eff}}}{\ln 2} / \mathrm{kg}\right)$, $n_{i}$ the number of emitted particles or photons per decay,

$\bar{E}_{i}$ the average energy of the emitted radiation,

$\Phi_{i}$ the part of the radiation absorbed in the organ, and

$T_{\text {eff }}$ the effective half-life of the radioisotope in the organ.

Several computerized versions of the MIRD formalism, e.g. MIR-DOSE (a PC-based program) [3], are available. The dose calculations help to decide whether the radioisotope under study is suitable for diagnostic applications. The underlying principle in all diagnostic investigations is that the radiation dose to the patient should be as low as possible.

A second important aspect of radioactive decay data is related to the detection of the emitted radiation. With the everincreasing significance of emission tomography, viz. Single Photon Emission Computed Tomography (SPECT) and Positron Emission Tomography (PET), radioisotopes emitting suitable radiation are in great demand. As regards therapeutic radioisotopes, a high radiation dose is deliberately desired. For an accurate dose estimation, however, good radiation detection possibility is required. With pure $\beta^{-}$-emitters (e.g. ${ }^{32} \mathrm{P},{ }^{90} \mathrm{Y}$, etc.) the distribution of radioactivity cannot be measured from outside the body. The dosimetry in the relevant applications is therefore rather empirical. The decay data are thus of great significance in the choice and application of a radioisotope in medicine. Some of the important types of relevant decay data are given in Table 1 .

In general, the nuclear structure and decay data are well known, and considerable efforts have been harnessed to establish data files and libraries [4-6]. As far as medical applications are concerned, the MIRD Committee has compiled data for about 250 radionuclides, including decay schemes and equilibrium dose constants [7]. In fact these data are the basis for further nuclear medical work. However, it is not excluded that a radioisotope finds considerable use, although some of its decay characteristics are not known well. Some of the areas where deficiencies may exist include $\beta^{-}$- and $\gamma$-ray intensities, EC- and $\beta^{+}$-decay branching, Auger electron spectra and intensities, etc. All those deficiencies lead to some inaccuracy in the estimation of radiation dose and quantitation of effect of ionising radiation.

Nuclear theory and systematics are sufficiently advanced to predict decay data for normal nuclei with reasonable accuracies. Only for light nuclei and for those far away from the stability line the predictions are less certain. Still, in recent years, two developing areas of nuclear medicine have revealed some deficiencies in the existing decay data. One pertains to endoradiotherapy (and brachytherapy) with radioisotopes emitting soft radiation, such as X-rays, Auger electrons and low-energy $\beta^{-}$- or $\beta^{+}$-particles. The other one pertains to PET studies with non-common longer-lived $\beta^{+}$-emitters. Accurate decay data (e.g. energies and intensities of emitted radiation) can only be obtained if the radioisotope under investigation is available in a pure form (through the choice of a proper production reaction and chemical or mass separation). A recent example is furnished by the potentially useful $\beta^{+}$-emitter ${ }^{120 \mathrm{~g}} \mathrm{I}\left(T_{1 / 2}=1.35 \mathrm{~h}\right)$. Its $\beta^{+}$-decay branching value was reported to be between 39 and $81 \%$. Recent radiochemical studies [8] established a value of $56 \pm 3 \%$. Thus, despite the huge body of decay data available, it may be occasionally necessary to perform new and accurate measurements on some selected radioisotopes.

\section{Nuclear reaction data}

These data are needed in radioisotope production programmes, mainly for optimisation of production routes. Since radioisotopes are produced in reactors as well as at cyclotrons, both neutron and charged particle induced reaction cross section data are required. The energy ranges involved are rather broad. In the case of neutrons mostly thermal en-
Table 1. Nuclear data relevant to medical applications of radioisotopes.

\begin{tabular}{|c|c|c|}
\hline Type of data & Quantities & Main applications \\
\hline Radioactive decay data & $\begin{array}{l}\text { - Half-life } \\
-\alpha-, \beta-, \gamma \text {-ray energies and } \\
\text { intensities } \\
\text { - EC } / \beta^{+} \text {branching ratio } \\
\text { - IT, conversion coefficient } \\
\text { - X-ray and Auger electron } \\
\text { intensities } \\
\text { - Decay scheme }\end{array}$ & $\begin{array}{l}\text { Choice of the radioiso- } \\
\text { tope for medical use } \\
\text { (diagnosis, therapy) } \\
\text { - Internal radiation dose } \\
\text { calculation }\end{array}$ \\
\hline \multirow[t]{4}{*}{ Nuclear reaction data } & Neutron data & \\
\hline & $\begin{array}{l}\text { - Scattering and capture } \\
\text { cross sections } \\
\text { - Resonance integrals } \\
\text { - (n, charged particle) } \\
\text { reaction cross sections } \\
\text { - Fission yields }\end{array}$ & $\begin{array}{l}\text { - Reactor production of } \\
\text { radioisotopes }\end{array}$ \\
\hline & Charged particle data & \\
\hline & $\begin{array}{l}\text { - Reaction thresholds } \\
\text { - Excitation functions } \\
\text { - Differential and integral } \\
\text { data } \\
\text { - Thin and thick target } \\
\text { yields }\end{array}$ & $\begin{array}{l}\text { - Cyclotron production of } \\
\text { radioisotopes }\end{array}$ \\
\hline
\end{tabular}


ergies and fission neutron spectrum are important, and in charged particles, energy ranges extending from a few $\mathrm{MeV}$ up to several hundred $\mathrm{MeV}$. A summary of the reaction cross section data needed in radioisotope production programmes is given in Table 1 . We briefly outline the importance of nuclear data in meeting some of the quality requirements of the product.

In reactor production of radioisotopes, the most commonly used nuclear routes include (n, $\gamma$ ), (n,fission) and (n,charged particle) processes. The $(\mathrm{n}, \gamma)$ reaction has generally a high cross section at thermal neutron energies, so that the yield of the product is rather high. However, a serious drawback of the process is the low specific radioactivity which makes the radioisotope less suitable for medical applications. The specific radioactivity can be improved through various methods (cf. Qaim, this issue, contribution on diagnostic radioisotopes). Nonetheless, in principle the drawback remains. The fission process is a very suitable method to produce a large number of radioisotopes in a "nocarrier-added" form. The chemical processing involved, however, is rather extensive. The $(n, p)$ and $(n, \alpha)$ reaction cross sections are generally low; these processes are therefore used to produce only a few radioisotopes in the light mass element region. In general the nuclear data needed in radioisotope production programmes are of integral nature (e.g. fission neutron spectrum averaged cross sections) and are mostly available in the evaluated neutron data libraries.

The cross section data base for the reactor production of radioisotopes is well established. Only for some special radioisotopes under development (e. g. those produced via double neutron capture), new measurements and evaluations may be necessary. As far as the predictive power of nuclear theory and systematics is concerned, the low-energy (n, $\gamma$ ), $(\mathrm{n}, \mathrm{p})$ and $(\mathrm{n}, \alpha)$ reactions are described reasonably well by the statistical model, and the fission yields are predicted with good accuracies by the systematics.

In cyclotron production of radioisotopes, the reaction cross section data play a very important role (for early reviews [cf. 9, 10]). Due to rapid degradation of the projectile energy in the target material, the energy range covered within the target is relatively broad, and, since the reaction cross section varies rather rapidly with energy, it is not appropriate to adopt an average cross section over the whole energy range. One needs rather the full excitation function of the nuclear process to be able to calculate the yield with a reasonable accuracy. A further important point is the number of competing reaction channels. At an incident projectile energy of $20 \mathrm{MeV}$, for example, about six reaction channels with significant cross sections accur. With a view to estimating the activity balance, it is imperative to know the cross sections of all those processes. Production of radioisotopes may be carried out using protons, deuterons, ${ }^{3} \mathrm{He}-$ or $\alpha$-particles. A knowledge of all the reaction cross sections is necessary. The demands on the data may thus be extensive. In practice, however, only a few radioisotopes are produced via reactions induced by particles other than protons. The data requirements are therefore foreseeable. At small-sized cyclotrons, low-energy reactions like $(p, n)$, $(\mathrm{p}, \alpha),(\mathrm{d}, \mathrm{n}),(\mathrm{d}, \alpha)$, etc. are used. At higher energies, on the other hand, $(\mathrm{p}, \mathrm{xn})$ reactions are commonly employed. In some special cases, the (p,spall) process is applied.
From a given excitation function, the expected yield of a product for a certain energy range (i.e. target thickness) can be calculated using the expression:

$$
Y=\frac{N_{\mathrm{L}} \cdot H}{M} I\left(1-\mathrm{e}^{-\lambda t}\right) \int_{E_{1}}^{E_{2}}\left(\frac{\mathrm{d} E}{\mathrm{~d}(\rho x)}\right)^{-1} \sigma(E) \mathrm{d} E,
$$

where $N_{\mathrm{L}}$ is the Avogadro number, $H$ the enrichment (or isotopic abundance) of the target nuclide, $M$ the mass number of the target element, $I$ the projectile current, $\left(\frac{\mathrm{d} E}{\mathrm{~d}(\rho x)}\right)$ the stopping power, $\sigma(E)$ the cross section at energy $E, \lambda$ the decay constant of the product and $t$ the time of irradiation. The calculated yield value represents the maximum yield which can be expected from a given nuclear process.

The optimisation of a nuclear process for the production of a radioisotope at a cyclotron involves a selection of the projectile energy range that will maximise the yield of the product and minimise that of the radioactive impurities. Whereas the non-isotopic impurities produced can be removed by chemical separations, the level of isotopic impurities can be suppressed only using enriched isotopes as target materials and/or by a careful selection of the particle energy range effective in the target.

The radioisotope ${ }^{123} \mathrm{I}\left(T_{1 / 2}=13.2 \mathrm{~h}\right)$, a commonly employed halogen nuclide for labelling biomolecules for diagnostic studies using SPECT, for example, can be produced via various routes. Its production via the ${ }^{124} \mathrm{Te}(\mathrm{p}, 2 \mathrm{n})^{123} \mathrm{I}$ reaction furnishes a good example of the importance of nuclear data. In order to decrease the level of isotopic impurities in the ${ }^{123} \mathrm{I}$ produced, it is essential to use highly enriched ${ }^{124} \mathrm{Te}$ as target material. However, due to the competing ${ }^{124} \mathrm{Te}(\mathrm{p}, \mathrm{n})^{124} \mathrm{I}$ reaction it is not possible to eliminate the ${ }^{124} \mathrm{I}$ impurity completely, even if ${ }^{124} \mathrm{Te}$ is $100 \%$ enriched. Fig. 1, based on the excitation function measurements described in $[11,12]$, shows that the suitable proton energy range for the production of ${ }^{123} \mathrm{I}$ is $E_{\mathrm{p}}=25.0 \rightarrow 18.0 \mathrm{MeV}$, i.e. the energy of the incident protons should be selected as $25.0 \mathrm{MeV}$ and the thickness of the tellurium target should degrade the incident energy only to $18.0 \mathrm{MeV}$. Under these conditions the yield of ${ }^{123} \mathrm{I}$ at the end of bombardment (EOB) amounts to $565 \mathrm{MBq} / \mu \mathrm{Ah}$ and the level of ${ }^{124}$ I impurity in ${ }^{123} \mathrm{I}$ to about $1 \%$. If still a lower impurity level is desired, the energy window would have to be further adjusted. Evidently,

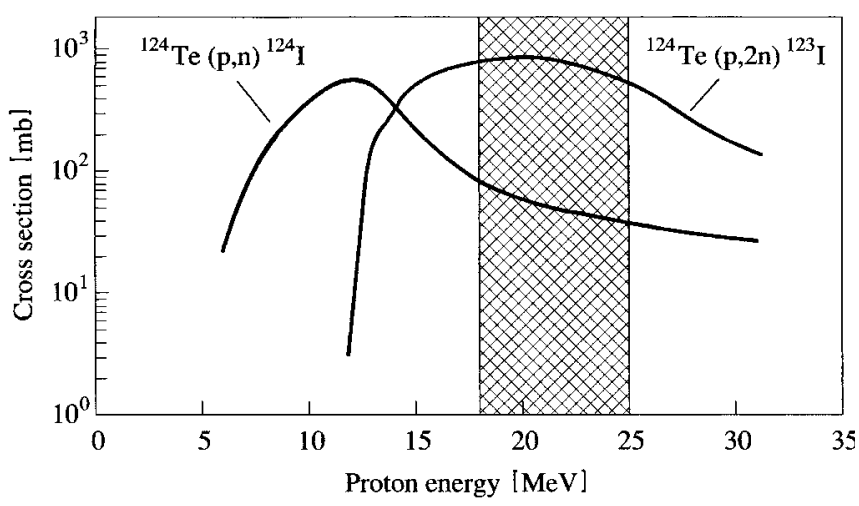

Fig. 1. Excitation functions of ${ }^{124} \mathrm{Te}(\mathrm{p}, \mathrm{n}){ }^{124} \mathrm{I}$ and ${ }^{124} \mathrm{Te}(\mathrm{p}, 2 \mathrm{n}){ }^{123} \mathrm{I}$ reactions. The suitable energy range for the production of ${ }^{123} \mathrm{I}$ is $E_{\mathrm{p}}=$ $25.0 \rightarrow 18.0 \mathrm{MeV}$ (based on data reported in $[11,12]$ ). 
it is necessary to know the excitation functions of the various competing reactions accurately.

The radioactive impurities have a dual effect: firstly, they adversely affect the line spread function in imaging and secondly, cause enhanced radiation dose to the patient. If the amount of a longer-lived impurity is too high, it may jeopardise the whole advantage of the short-lived radioisotope used. It may then be necessary to look for an alternative route of production for the desired radioisotope.

Besides isotopic impurities discussed above, in recent years the awareness of isomeric impurities has also increased. Several medically interesting research type radioisotopes have isomeric states which are rather disturbing. A few examples are ${ }^{94 \mathrm{~m}} \mathrm{Tc}\left({ }^{94 \mathrm{~g}} \mathrm{Tc}\right),{ }^{120 \mathrm{~g}} \mathrm{I}\left({ }^{120 \mathrm{~m}} \mathrm{I}\right)$, etc. The isomeric impurities cannot be controlled through a careful adjustment of the energy window (as mentioned above). Since the isomeric cross section ratio is primarily dependent on the type of reaction involved (for a review see [13]), it is essential to investigate all the possible production routes and then to choose the reaction and the energy range giving the best results. Obviously, nuclear data play here a very important role.

The nuclear reaction cross section data relevant to the production of the commonly used cyclotron radioisotopes are fairly well known, and a recent IAEA-TECDOC [14], prepared as an outcome of a Coordinated Research Programme (CRP) on "Medical Radioisotope Production", describes the recommended data in the desired energy ranges. New measurements are generally required while searching for an alternative route of production of a useful radioisotope or while performing development work on a potentially important radioisotope. The two fast developing areas of nuclear medicine mentioned above, viz. endoradiotherapy and PET studies using longer-lived $\beta^{+}$-emitters, demand considerable amount of experimental nuclear data work.

As far as nuclear model calculations are concerned, in the case of charged particle induced reactions, varying degree of success has been met. The cross sections for the light mass target nuclei can hardly be described by the model calculations. In the medium and heavy mass regions, however, both the compound/precompound and precompound/hybrid models (using, for example, the codes STAPRE/GNASH and ALICE-IPPE, respectively) have been partially successful. In general, a priori calculations do not meet the accuracy requirements needed in radioisotope production programmes. Experimental studies are thus often necessary.

\section{Significance of nuclear data in external radiation therapy}

External radiation therapy involves the use of electrons, $\mathrm{X}$-rays, high-energy $\gamma$-rays or hadrons (neutrons, protons, heavy ions, etc.). The use of low-energy electrons (emitted from radioactive sources or supplied by small accelerators), $\mathrm{X}$-rays (from conventional sources), high-energy electrons (supplied by a LINAC) and high-energy photons (produced as bremsstrahlung in the interactions of high-energy electrons with heavy elements) constitutes conventional or standard therapeutic practice. The interactions involved represent well-understood phenomena in atomic physics, and the data needed for both electron and photon therapy are well documented. Only in the case of very high energy photons, photonuclear processes like $(\gamma, n),(\gamma, 2 n)$, etc. may occur. The energy and angular distributions of the emitted neutrons are then of importance both from the viewpoint of radiation transport and radiation shielding. Furthermore, some activation products may also be formed.

Two important considerations in radiation therapy are the linear energy transfer (LET) and the relative biological efficiency (RBE). Use of hadrons in comparison to conventional therapy can have the advantage of a better physical selectivity, i.e. an improved dose profile and a higher biological efficiency, corresponding to greater killing in the tumour [cf. 15]. However, the various hadrons differ in their properties. In Fig. 2 the depth-dose relationship of photons, neutrons, protons and nitrogen ions are compared. Obviously neutrons and photons have a similar dose distribution whereas for charged particles the dose increases with the penetration depth, reaching a maximum value in the Braggpeak area. Neutrons do not exhibit this advanced physical dose profile but differ in their RBE from photons. The ultimate aim of hadron therapy is to optimise both physical and biological selectivity (cf. contributions by Jones and by Wambersie, on the rationale of hadron therapy, this issue).

In ion therapy, both atomic and nuclear interactions are involved, the contribution of the latter increasing with increasing energy. The interactions of neutrons are somewhat difficult to understand since they involve many nuclear phenomena which cannot always be calculated in a reliable manner.

In neutron therapy, cross sections are needed for the formation of secondary neutrons, $\gamma$-rays and charged particles; a very brief summary is given in Table 2 . The data are required to estimate the absorbed dose in the patient. Information on secondary neutrons and $\gamma$-rays is needed to calculate radiation transport over large distances. The data on charged

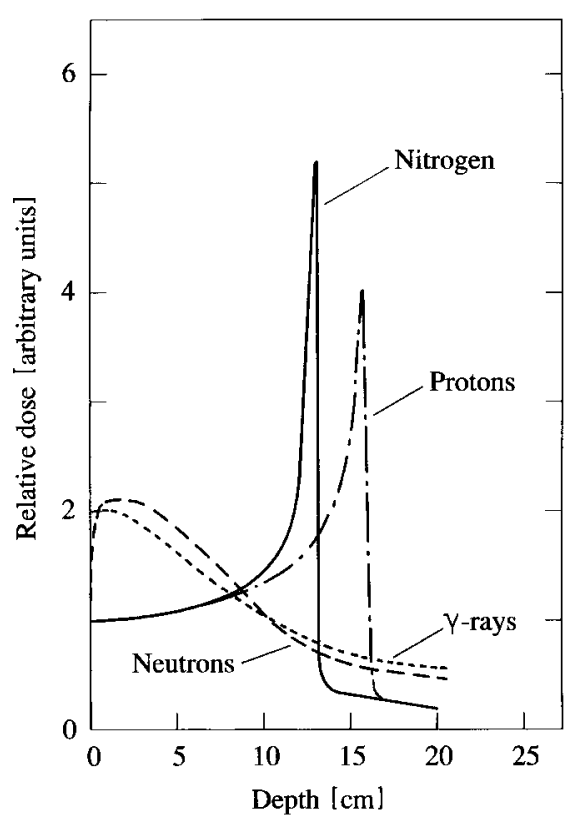

Fig. 2. Depth dose distribution of $\gamma$-rays, neutrons, protons and nitrogen ions in tissue (adapted from [15]). The area around the maximum dose in the case of charged particles is termed as "Bragg-peak" area. 
Table 2. Nuclear data relevant to hadron therapy.

\begin{tabular}{|c|c|c|}
\hline Type of data & Quantities & Main applications \\
\hline Neutron data & $\begin{array}{l}\text { - Elastic and nonelastic } \\
\text { cross sections } \\
\text { - Secondary neutron and } \\
\gamma \text {-ray emission (differen- } \\
\text { tial and double differential } \\
\text { cross sections) } \\
\text { - Charged particle (p, } \mathrm{d}, \mathrm{t}, \alpha) \\
\text { emission (differential and } \\
\text { double differential cross } \\
\text { sections) } \\
\text { - Activation cross sections }\end{array}$ & $\begin{array}{l}\text { - Calculation of neutron } \\
\text { spectra } \\
\text { - Radiation transport calcu- } \\
\text { lations } \\
\text { - Calculation of kerma } \\
\text { coefficients } \\
\text { - Determination of activity } \\
\text { balance }\end{array}$ \\
\hline Proton data & $\begin{array}{l}\text { - Total nonelastic cross } \\
\text { sections } \\
\text { - Neutron, charged particles } \\
\text { and } \gamma \text {-ray emission } \\
\text { (differential and double } \\
\text { differential cross sections) } \\
\text { - Average energies for the } \\
\text { light ejectiles } \\
\text { - Product recoil spectra } \\
\text { - Activation cross sections }\end{array}$ & $\begin{array}{l}\text { - Estimation of proton } \\
\text { beam depletion } \\
\text { - Calculation of energy } \\
\text { deposition and radiation } \\
\text { transport }\end{array}$ \\
\hline
\end{tabular}

particle emission in fast neutron induced reactions are of considerable interest for calculating the heat deposited in the tissue. The effect is expressed in terms of kerma coefficient (kinetic energy released in matter). The accuracy of the neutron induced cross sections is often estimated by a comparison of kerma coefficients derived from cross sections with those obtained experimentally. If the cross sections give a kerma coefficient that is in agreement with experimental measurements, the confidence in the absorbed dose predictions by a transport code is increased.

In proton therapy, cross sections are needed for the formation of neutrons and $\gamma$-rays, and to some extent secondary charged particles. This information is required to calculate the radiation transport and radiation dose. A brief summary of the data needed is given in Table 2. The major advantage of this therapy is the capability to treat deep-lying tumours, close to critical structures. This is possible mainly due to the high selectivity of the Bragg-peak.

The heavy-ion beam therapy has been performed with ${ }^{12} \mathrm{C},{ }^{14} \mathrm{~N},{ }^{20} \mathrm{Ne},{ }^{28} \mathrm{Si}$ and ${ }^{40} \mathrm{Ar}$ ions, but currently only ${ }^{12} \mathrm{C}$ is used. However, this is a rather specialized technique and, despite its great potential, has found so far only limited application. Another therapeutic approach is represented by the administration of a boron based compound and, after its accumulation in the tumour containing organ, irradiation via an external thermal (better epithermal) neutron source (boron neutron capture therapy, BNCT). The nuclear process ${ }^{10} \mathrm{~B}(\mathrm{n}, \alpha){ }^{7} \mathrm{Li}$ leads to the release of short-range, densely ionising $\alpha$-particles and the ${ }^{7} \mathrm{Li}$-recoils which cause the therapeutic effect.

\section{Spectral data}

From the above discussion it is evident that in radiation therapy primarily spectral data are needed. Besides total, elastic and nonelastic scattering cross sections, the energy- and angle-dependent emission spectra of $\gamma$-rays, neutrons and charged particles are needed. Due to the large effort involved in such studies, only a limited number of experimental data sets for biologically important target elements are available. A heavy reliance on theory is therefore obvious. Since the accuracy requirements are not as stringent as in the case of data for production of diagnostic radioisotopes, the data predicted via nuclear model calculations are generally acceptable (cf. Chadwick, this issue).

The availability of medium-energy nuclear data for radiation therapy has been discussed (cf. Haight, this issue). The new needs arising in connection with newly emerging therapies are under constant review [cf. 16, 17]. Recently a very thorough group study on nuclear data for neutron and proton radiotherapy has been performed [18] under the auspices of the International Commission on Radiation Units and Measurements. The final report of the group [18] gives information on neutron-induced nuclear reaction cross sections and kerma coefficients up to $150 \mathrm{MeV}$, and proton-induced cross sections up to $250 \mathrm{MeV}$. The energy range of neutrons currently used in therapy extends to about $70 \mathrm{MeV}$. However, since higher energies may be used in the future, the data are given up to $150 \mathrm{MeV}$. All the data were obtained by a combination of experiment and theory. For this purpose, use was made of the GNASH nuclear model code, which incorporates theories for compound nucleus, preequilibrium and direct interactions. Optical model calculations served to determine total, total nonelastic and elastic scattering cross sections.

In hadron therapy nuclear data are needed primarily for the major isotopes of $\mathrm{H}, \mathrm{C}, \mathrm{N}, \mathrm{O}, \mathrm{Al}, \mathrm{Si}, \mathrm{P}, \mathrm{Ca}, \mathrm{Fe}$, $\mathrm{Cu}, \mathrm{W}$ and $\mathrm{Pb}$, the latter elements being collimator and shielding materials. Most detailed information is needed for the most important elements, with lesser information for the others. The above mentioned report [18] follows this strategy. 


\section{Activation products}

In general, the activation of tissue components is not very serious and hence very few experimental studies have been reported with regard to the formation of activation products in neutron as well as proton therapy. In several special cases, however, activation may not be negligible. We discuss some of them below.

The interactions of fast neutrons with light mass nuclei lead to the formation of significant amounts of tritium $\left(T_{1 / 2}=12.3 \mathrm{a}\right)$. In a comprehensive study with $53 \mathrm{MeV}$ $\mathrm{d}(\mathrm{Be})$ neutrons it was shown [19] that the $(\mathrm{n}, \mathrm{t})$ cross sections for the lightest elements are exceptionally large (between 10 and $400 \mathrm{mb}$ ). However, for elements with $Z>20$ the triton emission cross section is constant at about $5 \mathrm{mb}$. Another activity observed was ${ }^{7} \mathrm{Be}\left(T_{1 / 2}=53.3 \mathrm{~d}\right)$; however, with the cross section in the $\mu \mathrm{b}$ range [20]. In the medium mass region several short- and long-lived products were also observed with formation cross sections of 5 to $10 \mathrm{mb}[19,21]$. Thus, except for tritium, the radioactive products formed in neutron therapy may not be of much significance. The activation of shielding and collimator materials, however, may be significant.

Comprehensive experimental studies on the formation of activation products in proton induced reactions exist in the literature [22-24]. However, the biologically relevant elements have not been investigated in detail. As regards proton therapy, a few experimental studies have been performed in the following four directions:

a) formation of short-lived $\beta^{+}$-emitters, like ${ }^{11} \mathrm{C}\left(T_{1 / 2}=\right.$ $20.3 \mathrm{~min}),{ }^{13} \mathrm{~N} \quad\left(T_{1 / 2}=10.0 \mathrm{~min}\right) \quad$ and ${ }^{18} \mathrm{~F} \quad\left(T_{1 / 2}=\right.$ $110.0 \mathrm{~min})$,

b) accumulation of ${ }^{7} \mathrm{Be}\left(T_{1 / 2}=53.3 \mathrm{~d}\right)$,

c) formation of medium mass activation products, like ${ }^{22} \mathrm{Na}$ $\left(T_{1 / 2}=2.6 \mathrm{a}\right),{ }^{42} \mathrm{~K}\left(T_{1 / 2}=12.4 \mathrm{~h}\right),{ }^{48} \mathrm{~V} \quad\left(T_{1 / 2}=16.0 \mathrm{~d}\right)$, ${ }^{51} \mathrm{Cr}\left(T_{1 / 2}=27.7 \mathrm{~d}\right)$, etc.,

d) accumulation of long-lived activity in collimators, like ${ }^{54} \mathrm{Mn}\left(T_{1 / 2}=312.2 \mathrm{~d}\right)$, etc.

From those studies it was concluded that the amount of ${ }^{7} \mathrm{Be}$ accumulated is negligible [25]. Similarly the medium mass activation products are also not significant [26]. The activation of collimators, however, is appreciable [27] and due precautions are necessary to protect the therapy personnel. The formation of short-lived $\beta^{+}$-emitters is of considerable scientific interest. We therefore discuss it in more detail.

The formation cross sections of short-lived $\beta^{+}$-emitters are significant but the information available over the whole energy range is rather scanty. The results of a recent measurement and analysis on the formation of ${ }^{11} \mathrm{C}\left(T_{1 / 2}=\right.$ $20.3 \mathrm{~min}$ ) from ${ }^{\text {nat }} \mathrm{N}$ are shown in Fig. 3 [28]. Whereas extensive data exist up to $20 \mathrm{MeV}$ [14], the data base at higher energies is rather weak. Worth mentioning is that two major contributing processes with different thresholds are involved, viz. ${ }^{14} \mathrm{~N}(\mathrm{p}, \alpha){ }^{11} \mathrm{C}$ and ${ }^{14} \mathrm{~N}(\mathrm{p}, 2 \mathrm{p} 2 \mathrm{n}){ }^{11} \mathrm{C}$. A small contribution from the ${ }^{15} \mathrm{~N}(\mathrm{p}, \alpha \mathrm{n}){ }^{11} \mathrm{C}$ reaction is also expected. A theoretical calculation based on the precompound hybrid code ALICE-IPPE, incorporating $\alpha$-particle emission, is also shown in Fig. 3. Obviously the theory reproduces the shape of the excitation function to some extent. However, the magnitudes differ considerably.

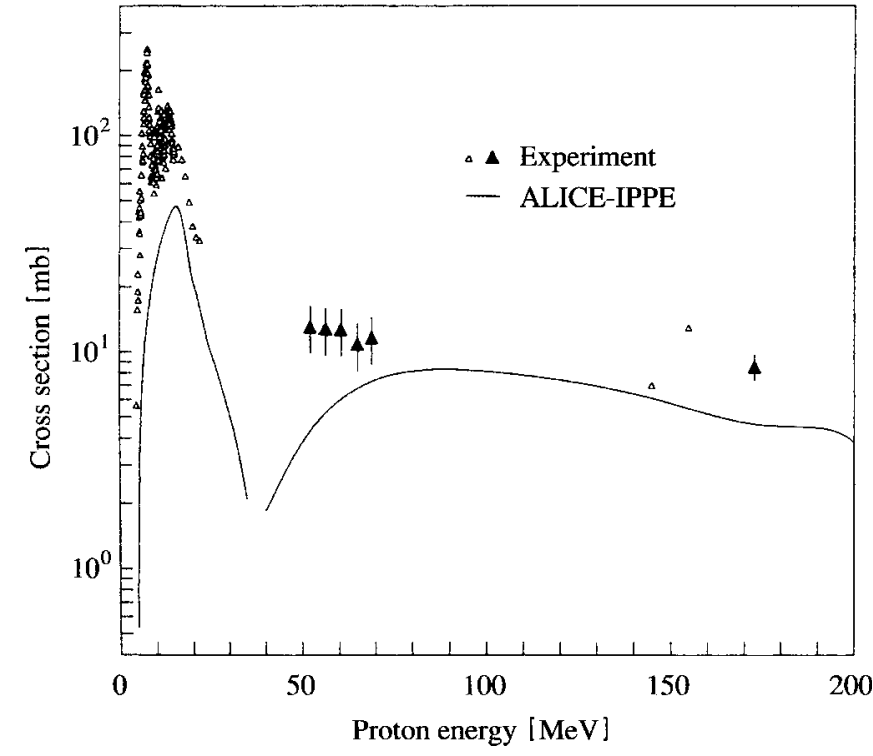

Fig. 3. Excitation function of the ${ }^{\text {nat }} \mathrm{N}(\mathrm{p}, \mathrm{x}){ }^{11} \mathrm{C}$ process. For a detailed compilation of the data up to $20 \mathrm{MeV}$, see [14]. The diagram has been adapted from [28].

The cross section data for the formation of short-lived $\beta^{+}$-emitters are important from two viewpoints: firstly, for estimating the extra dose caused to the patient, and secondly, for precise localisation of the dose distribution related to therapy. Regarding the first, an estimate was done [28] for liver as a source organ and a $200 \mathrm{MeV}$ proton beam intersity of $5 \mathrm{nA} / \mathrm{cm}^{2}$. The dose caused by ${ }^{11} \mathrm{C},{ }^{13} \mathrm{~N}$ and ${ }^{15} \mathrm{O}$ amounts to approximately $500 \mathrm{mSv}$. Compared to a total radiation dose of $2 \mathrm{~Sv}$ per therapy run, the enhancement in the radiation dose through the decay of the short-lived $\beta^{+}$-emitters amounts to about $25 \%$. Regarding the localisation of the dose distribution, a combination of PET and proton therapy is very promising [2]. Ideally, a simultaneous combination is desired. However, technically it is very difficult since it involves placing the therapy gantry and the PET machine very close to each other (around the patient). A PET investigation after the ion therapy (using ${ }^{11} \mathrm{C}$ formed during the therapy run) is more convenient and is practised at a few therapy centres.

In summary, the activation cross sections may not be of great significance in general radiation therapeutic applications. However, they are of considerable importance in following some special therapeutic aspects.

\section{Development of nuclear data file}

The development of a nuclear data file for applications involves several steps (Fig. 4). Experimental measurements are done using both activation and spectral methods. It is important that the reported data contain a full description of the experiment and various sources of errors together with their estimated magnitudes. The second source of data is the theory. Many nuclear models have been developed to predict unknown cross sections. Combining the experimental data and theoretical results to obtain the best values of cross sections is termed as "Evaluation". An evaluator attempts to derive "best" values from a set of incomplete experiments 


\section{Nuclear Data Development for Applications}

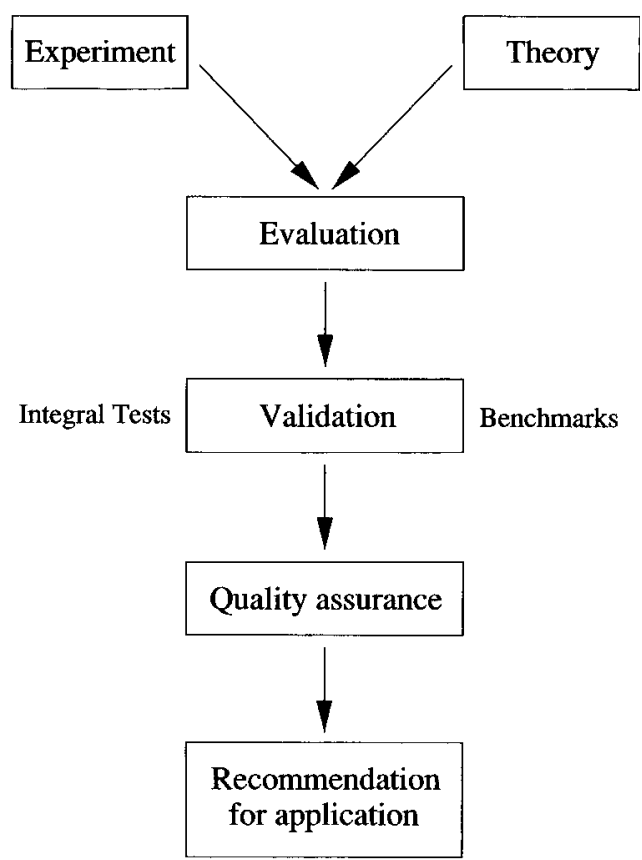

Fig. 4. Steps in the development of a nuclear data file for applications.

and only partially reliable theoretical models. In the exercise, often several adjustments have to be made. In energy regions where few measurements exist, either due to lack of facilities or due to experimental difficulties, a heavy reliance on theory is called for. The next step involves the validation of evaluated data. This is generally done via some benchmarks or integral tests. If large deviations occur, some new measurements or calculations may be necessary. If the validation experiments exhibit good reliability, some quality assurance tests, especially a description of errors and definition of application limits are essential. The data thus obtained are then considered as recommended data and constitute a "file" for some special application.

Many types of data files exist in the literature and are coordinated by one or more of the four major Nuclear Data Centres, namely,

- NNDC, Brookhaven, USA,

- OECD-NEA Data Bank, Paris, France,

- IAEA Nuclear Data Section, Vienna, Austria,

- Nuclear Data Centre, Obninsk, Russia.

However, the majority of those files deal with the energy related data. As far as medical applications are concerned, the efforts invested so far have been rather modest, mostly directed to neutron therapy. The evaluation methodology (especially for data related to diagnostic applications) is hitherto not as developed as in the case of energy related data. The two most recent comprehensive efforts, one related to diagnostic radioisotopes [14] and the other dealing with the hadron therapy [18], are expected to fill the existing gaps in data. Further detailed efforts are, however, essential to enhance our knowledge about several aspects of diagnosis and therapy.

\section{References}

1. Stöcklin, G., Qaim, S. M., Rösch, F.: The impact of radioactivity on medicine. Radiochimica Acta 70/71, 249 (1995).

2. Qaim, S. M.: Radioactivity in medicine: achievements, perspectives and role of nuclear data. Proc. Int. Conf. Nuclear Data for Science and Technology. Trieste, Italy, May 1997 (Reffo, G., Ventura, A., Grandi, C., Eds.), Italian Physical Society 59, 31 (1997).

3. Stabin, M.: MIR-DOSE 2 Program. Oak Ridge National Laboratory. Oak Ridge, Tennessee, USA (1985).

4. Pfennig, G., Klewe-Nebenius, H., Seelmann-Eggebert, M.: Karlsruher Nuklidkarte. Forschungszentrum Karlsruhe, Germany (1995).

5. Browne, E., Firestone, R. B.: Table of Radioactive Isotopes. (Shirley, V. S., Ed.), Wiley, London (1986).

6. Nuclear Data Sheets, Periodic issues.

7. Weber, D. A., Eckerman, K. G., Dillman, L. T., Ryman, J. C.: MIRD-Radionuclide Data and Decay Schemes. Society of Nuclear Medicine, New York (1989).

8. Hohn, A., Coenen, H. H., Qaim, S. M.: Positron emission intensity in the decay of ${ }^{120 g}$ I. Radiochimica Acta 88, 139 (2000).

9. Qaim, S. M.: Nuclear data relevant to cyclotron produced shortlived medical radioisotopes. Radiochimica Acta 30, 147 (1982).

10. Qaim, S. M.: Medical radioisotopes and nuclear data. Proc. IAEAConsultants' Meeting on Data Requirements for Medical Radioisotope Production. Tokyo, Japan, April 1987 (Okamoto, K., Ed.), Report INDC(NDS)-195, IAEA, Vienna (1988), p. 25.

11. Kondo, K., Lambrecht, R. M., Wolf, A. P.: ${ }^{123}$ I production for radiopharmaceuticals XX. Excitation functions of the ${ }^{124} \mathrm{Te}(\mathrm{p}, 2 \mathrm{n})^{123} \mathrm{I}$ and ${ }^{124} \mathrm{Te}(\mathrm{p}, \mathrm{n})^{124} \mathrm{I}$ reactions and the effect of target enrichment on radionuclidic purity. Int. J. Appl. Radiat. Isot. 28, 395 (1977).

12. Scholten, B., Kovács, Z., Tárkányi, F., Qaim, S. M.: Excitation functions of ${ }^{124} \mathrm{Te}(\mathrm{p}, \mathrm{xn}){ }^{124,123} \mathrm{I}$ reactions from 6 to $31 \mathrm{MeV}$ with special reference to the production of ${ }^{124} \mathrm{I}$ at a small cyclotron. Appl. Radiat. Isot. 46, 255 (1995).

13. Qaim, S. M.: Recent developments in the study of isomeric cross sections. Proc. Int. Conf. Nuclear Data for Science and Technology. Gatlinburg, Tennessee, USA, May 1994 (Dickens, J. K., Ed.), American Nuclear Society, La Grange Park, Illinois (1994), p. 186.

14. Gul, K., Hermanne, A., Mustafa, M. G., Nortier, F. M., Oblozinsky, P., Qaim, S. M. (Chairman), Scholten, B., Shubin, Y., Takács, S., Tárkányi, F. T., Zhuang, Y.: Charged Particle Cross Section Database for Medical Radioisotope Production. IAEA-TECDOC 1211, Vienna (2001), pp. 1-284.

15. Kraft, G., Kraft-Weyrather, W., Taucher-Scholz, G., Scholz, M.: What kind of radiobiology should be done at a hadron therapy centre? Advances in Hadrontherapy. (Amaldi, U., Larsson, B., Lemoigne, Y., Eds.), Elsevier, Amsterdam (1997), p. 38.

16. Kocherov, N. P. (Compiler): Status of Data Needed for Radiation Therapy and Existing Developing Activities in Member States. IAEA-Consultants' Meeting, December 1996, Report INDC (NDS)-365, IAEA, Vienna (1997).

17. Broerse, J. J., DeLuca, Jr., P. M., Dietze, G., Haight, R. C., Hiraoka, T., Kawashima, K., Kocherov, N., Menzel, H. G., Olsson, N., Wambersie, A., White, R. M., Zoetelief, J.: Nuclear Data for Neutron Therapy: Status and Future Needs. IAEA-TECDOC-992, Vienna, December (1997).

18. Barschall, H. H., Chadwick, M. B. (Chairman), Jones, D. T. L., Meulders, J.P., Schumacher, H., Young, P.G.: Nuclear Data for Neutron and Proton Radiotherapy and for Radiation Protection. ICRU Report 63, Bethesda, March (2000).

19. Qaim, S. M., Wölfle, R.: Triton emission in the interactions of fast neutrons with nuclei. Nucl. Phys. A 295, 150 (1978).

20. Scholten, B., Qaim, S. M., Stöcklin, G.: A systematic study of $\left(\mathrm{n},{ }^{7} \mathrm{Be}\right)$ reactions on medium and heavy mass nuclei induced by $53 \mathrm{MeV}$ d(Be)-breakup neutrons. Radiochimica Acta 62, 107 (1993).

21. Qaim, S. M., Wu, C. H., Wölfle, R.: ${ }^{3} \mathrm{He}$-particle emission in fast neutron induced reactions. Nucl. Phys. A 410, 421 (1983).

22. Bodemann, R., Lange, H.-J., Leya, I., Michel, R., Schiekel, Th., Rösel, R., Herpers, U., Hofmann, H. J., Dittrich, B., Suter, M., Wölfli, W., Holmquist, B., Condé, H., Malmborg, P.: Production 
of residual nuclei by proton-induced reactions on $\mathrm{C}, \mathrm{N}, \mathrm{O}, \mathrm{Mg}, \mathrm{Al}$ and Si. Nucl. Instr. Meth. Phys. Res. B 82, 9 (1993).

23. Schiekel, Th., Sudbrock, F., Herpers, U., Gloris, M., Lange, H.-J., Leya, I., Michel, R., Dittrich-Hannen, B., Synal, H.-A., Suter, M., Kubik, P. W., Blann, M., Filges, D.: Nuclide production by proton induced reactions on elements $(6 \leq Z \leq 29)$ in the energy range from $200 \mathrm{MeV}$ to $400 \mathrm{MeV}$. Nucl. Instr. Meth. Phys. Res. B 114, 91 (1996).

24. Michel, R., Bodemann, R., Busemann, H., Daunke, R., Gloris, M., Lange, H.-J., Klug, B., Krins, A., Leya, I., Lüpke, M., Neumann, S., Reinhardt, H., Schnatz-Büttgen, M., Herpers, U., Schiekel, Th., Sudbrock, F., Holmquist, B., Condé, H., Malmborg, P., Suter, M., Dittrich-Hannen, B., Kubik, P. W., Synal, H.-A., Filges, D.: Cross sections for the production of residnal nuclides by low- and medium-energy protons from the target elements $\mathrm{C}, \mathrm{N}, \mathrm{O}, \mathrm{Mg}, \mathrm{Al}$, $\mathrm{Si}, \mathrm{Ca}, \mathrm{Ti}, \mathrm{V}, \mathrm{Mn}, \mathrm{Fe}, \mathrm{Co}, \mathrm{Ni}, \mathrm{Cu}, \mathrm{Sr}, \mathrm{Y}, \mathrm{Zr}, \mathrm{Nb}, \mathrm{Ba}$ and $\mathrm{Au}$. Nucl. Instr. Meth. Phys. Res. B 129, 153 (1997).
25. Fassbender, M., Scholten, B., Qaim, S. M.: Radiochemical studies of $\left(\mathrm{p},{ }^{7} \mathrm{Be}\right)$ reactions on biologically relevant elements in the proton energy range of 50 to $350 \mathrm{MeV}$. Radiochimica Acta 81, 1 (1998).

26. Fassbender, H., Shubin, Yu. N., Qaim, S. M.: Formation of activation products in interactions of medium energy protons with $\mathrm{Na}$, Si, P, S, Cl, Ca and Fe. Radiochmica Acta 84, 59 (1999).

27. Fassbender, M., Shubin, Yu. N., Lunev, V. P., Qaim, S. M.: Experimental studies and nuclear model calculations on the formation of radioactive products in interactions of medium energy protons with copper, zinc and brass: Estimation of collimator activation in proton therapy facilities. Appl. Radiat. Isot. 48, 1221 (1997).

28. Fassbender, M., Scholten, B., Shubin, Yu. N., Qaim, S. M.: Activation cross section data for $(\mathrm{p}, \mathrm{x})$ processes of therapeutic relevance. Proc. Int. Conf. Nuclear Data for Science and Technology. Trieste, Italy, May 1997 (Reffo, G., Ventura, A., Grandi, C., Eds.), Italian Physical Society 59, 1646 (1997). 